\title{
Terahertz Spectra of Explosives Measured by Optical Parametric Oscillator-Based System and Time Domain Spectroscopy
}

\author{
N. Palka, T. Trzcinski* And M. Szustakowski \\ Institute of Optoelectronics, Military University of Technology, S. Kaliskiego 2, 00-908 Warsaw, Poland \\ In this paper, we report on measurements of absorption spectra of hexogen, penthrite, and octogen. The \\ measurements were carried out by means of time domain spectroscopy and compared to spectra obtained from a \\ setup, which bases on an optical parametric oscillator combined with a Golay cell. For time domain spectroscopy \\ measurement, Teraview TPS 3000 unit with accessories in transmission configuration was used.
}

PACS: 07.57.Ty, 78.20.Ci, 78.40.Pg, 42.65.Yj, 07.57.Kp

\section{Introduction}

We present the comparison of transmission spectra of three explosives (hexogen (RDX), penthrite (PETN), octogen (HMX)). The measurements were carried out by means of time domain spectroscopy (TDS) and compared to spectra obtained from a setup, which bases on an optical parametric oscillator (OPO) laser combined with a Golay cell. For TDS measurement, Teraview TPS 3000 unit with accessories in transmission configuration was used. The main parameters of the systems are: spectral range $0.06-3.5 \mathrm{THz}$, signal-to-noise better than 4000:1 and spectral resolution $0.06 \mathrm{THz}$. The chamber was purged with dry air to eliminate water vapor. The second setup consisted of commercially available tunable OPO from MSquared working in the range $0.7-2.2 \mathrm{THz}$ with repetition rate $53 \mathrm{~Hz}$, duration of the impulse of about $10 \mathrm{~ns}$ and energy $10 \mathrm{~nJ}$. The beam was detected by a Golay cell from Microtech using a lock-in amplifier.

Only small discrepancies between spectra measured by both methods are observed in the range $0.7-2.2 \mathrm{THz}$. For this range typical features of explosives can be identified using both methods. However, the TDS setup seems to have better performance in terms of signal-to-noise ratio and sensitivity.

\section{Sample preparation}

For the purpose of transmission considerations, we noted that pure materials have high attenuation, so we decided to mix them with polyethylene (PE) powders (matrix), which is highly transparent in the THz range.

The materials were ground using a mortar and pestle to reduce the particle size and to avoid scattering loss

\footnotetext{
* corresponding author; e-mail: ttrzcinski@wat.edu.pl
}

[1-3]. Next, the explosives were mixed with the matrix powder. The mixing ratio was $10 \%$ (weight of a material versus total weight of mixed samples). Afterwards, the sample was pressed into pellets (weight $400 \mathrm{mg}$ and $13 \mathrm{~mm}$ in diameter) directly using a hydraulic press. As a result we obtained the sample which consisted of $40 \mathrm{mg}$ of explosive material. As a reference, a pellet made of pure polyethylene with the same diameter and weight of $360 \mathrm{mg}$ was prepared.

\section{Time domain spectroscopy in transmission mode}

TDS [1-8] is a commonly used technique in THz range to measure transmission spectra of materials. In our measurements we used a commercially available spectrometer TPS 3000 from Teraview [9-11].

TDS setup with pulses generated by an $800 \mathrm{~nm}$ femtosecond Ti:sapphire laser (a pulse duration less than $100 \mathrm{fs}$, repetition rate of $80 \mathrm{MHz}$, and average power of $300 \mathrm{~mW}$ ) is presented in Fig. 1. The laser beam is split into a pump and probe and directed through a system of mirrors to an emitter and a detector, which bases on low temperature grown GaAs dipole antennas. The pump beam is focused on the biased emitter antenna to generate $\mathrm{THz}$ pulses through a photoconductive phenomenon. Such pulses last near $1 \mathrm{ps}$ and have broadband spectrum, usually in the range $0.1-3.0 \mathrm{THz}$ or more.

The transmitted $\mathrm{THz}$ beam can be detected by means of the detector antenna gated using the laser probe beam and a mechanical delay line. A lock-in amplifier and some software are used to collect and process data. The system is purged with dry air to eliminate water vapor.

This setup is commonly used for determination of transmission (or absorption) parameters of the materials. First step is to measure a reference - the pellet made of pure polyethylene in our case, which is inserted in the 


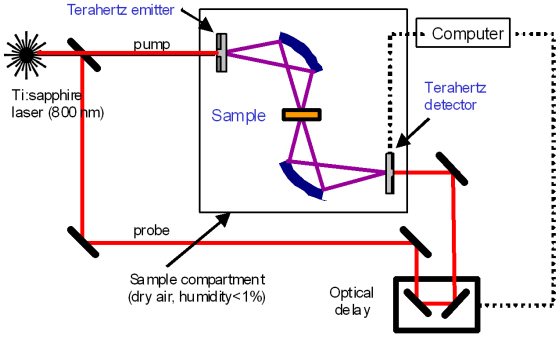

Fig. 1. Time domain spectrometry setup.

middle of the distance between the mirrors, perpendicularly to the incident $\mathrm{THz}$ beam. Next, the $\mathrm{THz}$ impulse for the pellet with the explosive material is determined in the same way (Fig. 2a). The transmission spectrum of the material is determined as the ratio between the power spectrum of the impulse of the pellet with material $\left(T_{\mathrm{M}}\right)$ to the power spectrum of the impulse of the reference $\left(T_{\mathrm{R}}\right)$. Figure 2a presents impulses measured for the reference and the RDX sample. A time shift between impulses is connected with the fact that samples with the explosive have slightly bigger refractive indices and thickness, which increases optical path inside the pellets with materials.
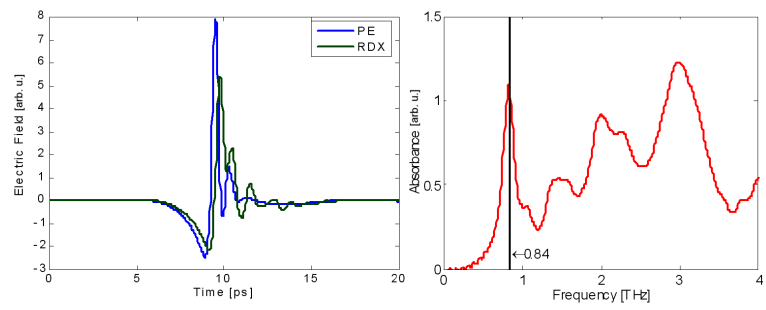

Fig. 2. THz impulses measured in the transmission configuration for reference (PE) and the RDX pellets (a) and absorbance of RDX (b).

It is a common practice in spectroscopic measurements to present data in logarithmic scale as absorption instead of transmission using absorbance $(A)$, which is defined as:

$$
A=-\log _{10}\left(T_{\mathrm{M}} / T_{\mathrm{R}}\right) \text {. }
$$

Figure $2 \mathrm{~b}$ shows absorbance of RDX sample determined by Eq. (1), respectively.

\section{OPO-based setup}

TDS is a mature and commonly used technique thanks to its high sensitivity and resolution. Hovewer, it suffers from the fact that the femtosecond laser, which is expensive, must be used for generation and detection of $\mathrm{THz}$ radiation.

On the other hand, scientific world is looking for another techniques, which can be applied for spectroscopic measurements. For comparison we tested a setup, which consists of the commercially available $\mathrm{THz}$ OPO laser Firefly from MSquared [12] and the very known and relatively cheap type of detector - the Golay cell.

OPO is a parametric oscillator that oscillates at optical frequencies. Its main task is to convert an input laser wave (called a "pump" wave) into two output waves of lower frequency ("signal" wave and "idler" wave) thanks to a nonlinear optical interaction in a usually crystalline gain medium. This phenomenon requires two conditions to be satisfied: the conservation of energy and momentum ("phase matching") [9, 10].

Figure 3 illustrates the scheme of the OPO cavity of Firefly laser. Pump light at $1064 \mathrm{~nm}$ from a $Q$-switched Nd:YAG laser incident onto the nonlinear crystal ( $\left.\mathrm{MgO}: \mathrm{LiNbO}_{3}\right)$ in the OPO cavity and is converted into two OPO output beams - a THz "signal" beam, and an "idler" beam. The "idler" beam varies in the range $1060-1080 \mathrm{~nm}$ according to the $\mathrm{THz}$ wavelength to which the laser is tuned. The nonlinear crystal is located at the intersection of the optical axis of the $\mathrm{Nd}$ :YAG laser cavity and the optical axis of the OPO idler wave cavity [12].

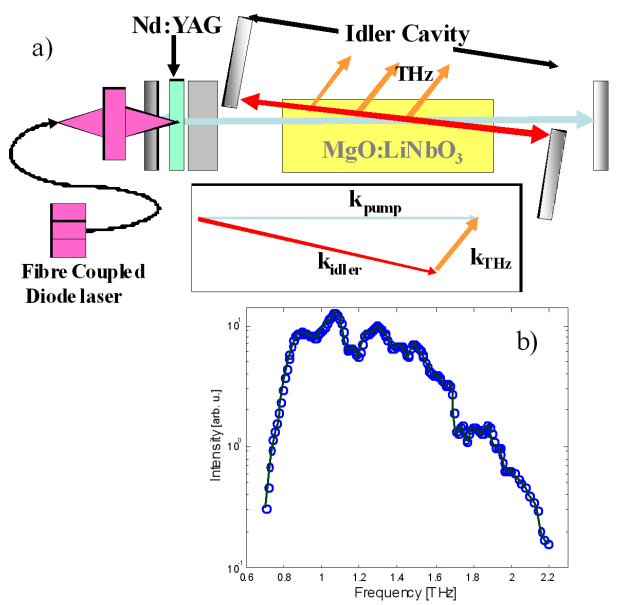

Fig. 3. Schematic illustration of Firefly laser with non co-linear, intersecting cavity configuration [11] (a) and its tuning curve (b).

The OPO cavity resonates only the idler and is therefore termed singly resonant at the idler frequency. The idler frequency is very close to the pump frequency because of the highly non co-linear intersecting and phase-matching geometry that is used. As may be seen from the cavity and the wave vector diagrams included in inset in Fig. 3, the frequency of the $\mathrm{THz}$ radiation can be tuned by changing the angle between the pump and OPO cavity axes. This angle is changed electro-mechanically and under computer control. Generating the $\mathrm{THz}$ radiation in cavity geometry also has the advantage that the $\mathrm{THz}$ beam has near-diffraction-limited spatial mode quality [12].

The laser can be tuned in the range $0.7-2.5 \mathrm{THz}$; its repetition rate equals to $53 \mathrm{~Hz}$, duration of the impulse 
is about $10 \mathrm{~ns}$, energy $10 \mathrm{~nJ}$ and spectral width $50 \mathrm{GHz}$. Tuning curve measured by means of Golay cell is presented in Fig. 3b. Due to poor signal intensity we limited our measurements to the range $0.7-2.2 \mathrm{THz}$.

Transmission measurements were performed in the system which is schematically shown in Fig. 4a. Terahertz radiation generated by the OPO laser is reflected from the gold-coated parabolic mirror $(f=10 \mathrm{~cm})$ and is focused on the detector - the Golay cell. We applied commercially available Golay cell from Microtech, which has the dynamic range: $100 \mathrm{nW}-1 \mathrm{~mW}$, the maximum modulation frequency $-50 \mathrm{~Hz}$ and the responsivity $10^{4} \mathrm{~V} / \mathrm{W}$ at $12.5 \mathrm{~Hz}$ modulation. As the repetition rate of the laser $(53 \mathrm{~Hz})$ is slight above the maximum modulation of the detector as well as the laser works in the impulse mode (impulse duration of about $10 \mathrm{~ns}$ ), we can obtain the signal whose amplitude is low $(\approx 50 \mathrm{mV}$ at maximum) and fluctuates in time - see Fig. 4b. Therefore, to cope with this problem and to gain higher dynamic range we applied the detection scheme which includes a lock-in amplifier and an averaging software.

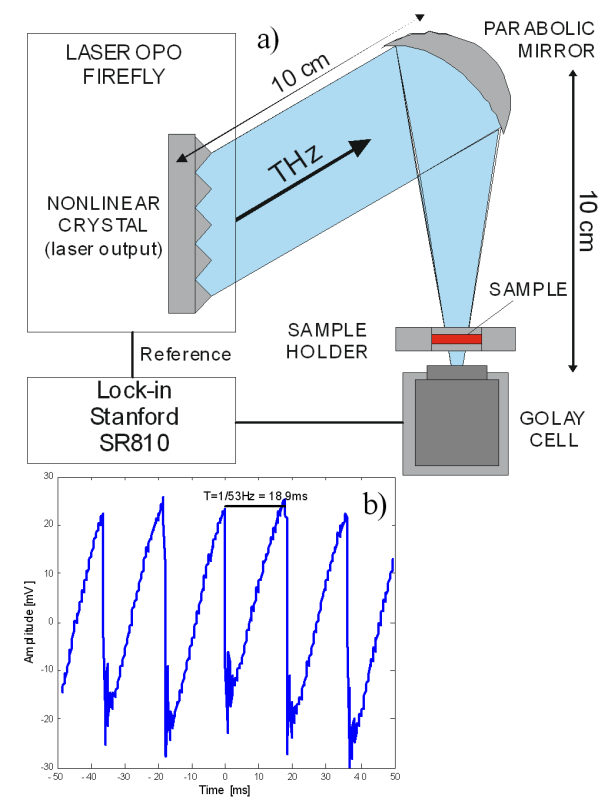

Fig. 4. OPO-based setup (a) and raw signal from the Golay cell (b).

The lock-in amplifier was connected via RS-232 to a computer with the LabView-based software. The signal from the laser was used as a reference. The signal from the lock-in was measured every $100 \mathrm{~ms}$ and averaged from 200 sequential measurements.

A sample holder was placed between the mirror and the detector. We used the same samples as for TDS measurements. Laser frequency was adjusted in the range $0.7-2.2 \mathrm{THz}$ with a step $0.01 \mathrm{THz}$. First, we measured the transmission for the reference pellet $\left(T_{\mathrm{R}}\right)$ and next - for the pellet with the explosive material $\left(T_{\mathrm{M}}\right)$. As for TDS, the transmission properties of the materials can be presented as transmission $\left(T_{\mathrm{M}} / T_{\mathrm{R}}\right)$ or absorbance $(A)$ using Eq. (1).

\section{Results}

Figure $5 \mathrm{a}-\mathrm{c}$ presents absorbance spectra of three commonly used explosives: RDX, PENT, and HMX measured by means of TDS (see Sect. 3) and the OPO-based setup (Sect. 4). TDS measurements were carried out in the range $0.1-3.5 \mathrm{THz}$, while OPO-based investigations are limited to the range $0.7-2.2 \mathrm{THz}$ due to the system performance. The spectra measured by two methods are in good conformity taking into account shape of the spectra and positions of characteristic peaks. The obtained main peaks of absorbance remain in good agreement with results of other groups [2-7].

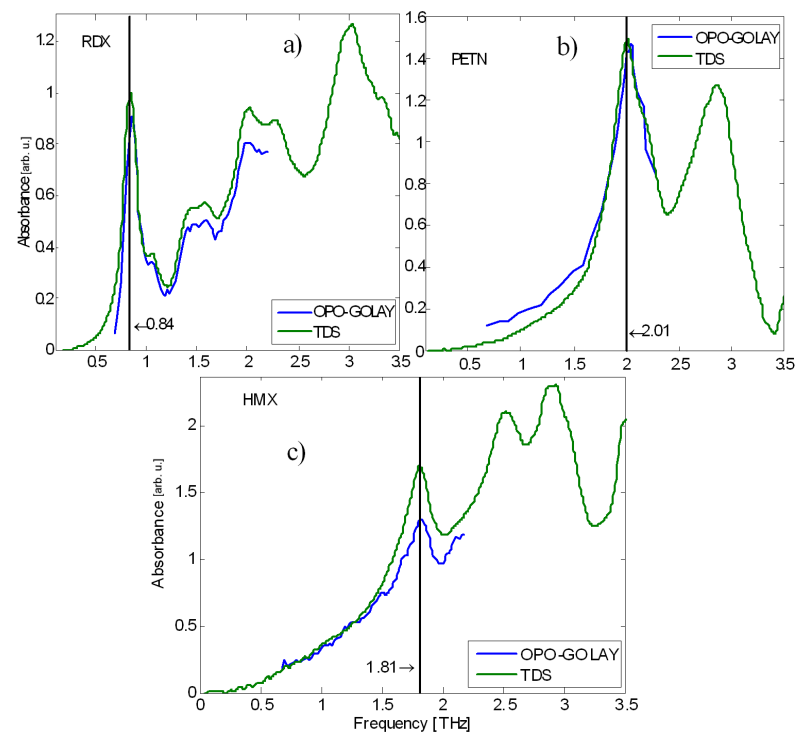

Fig. 5. Absorbances of RDX (a), PETN (b) and HMX (c) measure by TDS and the OPO-Golay setups.

\section{Summary}

To sum up, we measured and compared the terahertz transmission spectra of three commonly used explosive materials (RDX, HMX, PETN). We applied two techniques: time domain spectroscopy and the tuning optical parametric oscilator laser and the Golay cell detector.

It can be noted that TDS spectra are smooth and can be mesasured in the broadband range. This technique is fast (even 30 scans per second), mature and inherently very sensitive (dynamic range $\approx 70 \mathrm{~dB}$ in maximum).

On the other hand, spectra obtained from OPO-based system are rough. Since we applied the lock-in amplifier-based detection scheme and averaging, detection lasted even one hour to obtain good stability. We think that there could be a small change in frequency of laser during reference and material scanning and this can cause 
some fluctuations in transmission (or absorption) properties clearly seen in Fig. 5c at lower frequencies. Moreover, we used the Golay cell detector which is sensitive but slow to measure very fast impulses, which also can deteriorate the results. Therefore, in our future investigation we plan to use a fast and sensitive detector, which will consist of hot electron bolometer cooled to $4 \mathrm{~K}$.

\section{Acknowledgments}

The work was financed by the Polish Ministry of Science and Education under the project O N515 020140.

\section{References}

[1] L. Yun-Shik, Principles of Terahertz Science and Technology, Springer, New York 2008, p. 59.

[2] M.C. Kemp, IEEE Trans. Terahertz Sci. Technol. 1, $282(2011)$

[3] J. Chen, Y. Chen, H. Zhao, G.J. Bastiaans, X.-C. Zhang, Opt. Expr. 15, 12060 (2007).
[4] T. Pustelny, J. Ignac-Nowicka, B. Jarzabek, A. Burian, Opt. Appl. 34, 551 (2004).

[5] M.R. Leahy-Hoppa, M.J. Fitch, X. Zheng, L.M. Hayden, R. Osiander, Chem. Phys. Lett. 434, 227 (2007).

[6] D.J. Cook, B.K. Decker, M.G. Allen, Opt. Terahertz Sci. Technol. 6, 569 (2006).

[7] N. Palka, Acta Phys. Pol. A 120, 713 (2011).

[8] Teraview Ltd., Presentations and manuals, www . teraview .com (2011).

[9] D. Molter, M. Theuer, R. Beigang, Opt. Expr. 17, 6623 (2009).

[10] B. Pustelny, T. Pustelny, Acta Phys. Pol. A 116, 383 (2009).

[11] R. Sowade, I. Breunig, I.C. Mayorga, J. Kiessling, C. Tulea, V. Dierolf, K. Buse, Opt. Expr. 17, 22303 (2009).

[12] M Squared Lasers Ltd., Firefly-THz User Manual (2011). 